\title{
Metformin Attenuates Liver Fat Content: Finding from Schizophrenia Patients with Olanzapine-induced Weight Gain
}

\author{
Li Wang ${ }^{1, *}$, Yu Chen ${ }^{2, *}$, Yun-chuan Sui ${ }^{1}$, Xing-qi Tan ${ }^{3}$, Zhi Zhou ${ }^{4}$, Ning Li ${ }^{1}$, Le-ping $\mathrm{Xu}^{1}$ \\ ${ }^{1}$ Department of Psychiatry, ${ }^{3}$ Psychiatry Center, and ${ }^{4}$ Department of Radiology, No.102 Hospital of Chinese People's Liberation Army, Changzhou, \\ ${ }^{2}$ Department of Psychiatry, Affiliated Nanjing Brain Hospital of Nanjing Medical University, Nanjing, China
}

\begin{abstract}
Objective: This study was performed to evaluate the efficacy of metformin on liver fat content (LFC) in first episode schizophrenia patients with olanzapine-induced weight gain, and the relationship between the change of LFC and the other metabolic indices.

Methods: In a double-blind study, the clinically stable inpatients with first-episode schizophrenia under olanzapine monotherapy who gained more than $7 \%$ of their baseline weight were randomly assigned to two groups; one with olanzapine plus metformin $(1,000 \mathrm{mg} /$ day) (metformin group) and the other with olanzapine plus placebo (placebo group) for 16 weeks. All patients continued to maintain the original olanzapine dosage. LFC was measured by magnetic resonance imaging at baseline and at the end of 16 weeks, respectively. At the same time, glucose and lipid metabolism, homeostasis model assessment of insulin resistance index (HOMA-IR) were measured respectively, analyzing the correlation between the change value of LFC and other indicators.

Results: Over the 16-week study period, LFC value in metformin group decreased compared with baseline. LFC change across the 16-week treatment period was $-2.91 \%$ for the metformin group and $0.59 \%$ for the placebo group, with a between-group difference of $-3.5 \%$ (95\% confidence interval, -6.08 to $-0.93 ; p=0.009)$. Compared to baseline, in the metformin group, triglyceride and HOMA-IR reduced significantly, while high density lipoprotein cholesterol increased significantly at weeks 16 . There was positive correlation between LFC changes and triglycerides, HOMA-IR changes significantly.

Conclusion: Metformin can significantly attenuate LFC in schizophrenia patients with olanzapine-induced weight gain. It may be related to the improvement of the part of the glucolipid metabolic indices.
\end{abstract}

KEY WORDS: Insulin resistance; Metabolic syndrome; Metformin; Olanzapine; Schizophrenia; Liver fat.

\section{INTRODUCTION}

Currently, with the dramatic improvement achieved in clinical psychiatry by the use of atypical antipsychotic (AAP) agents, prevention and treatment of metabolic derangement induced by the AAP has gradually been a main concern in current clinical practice [1]. These AAP-associated adverse effects include weight gain, glucose dysregulation, insulin resistance, hyperlipidemia and hyper-

Received: February 20, 2019 / Revised: May 8, 2019

Accepted: May 9, 2019

Address for correspondence: Le-ping Xu

Department of Psychiatry, No.102 Hospital of Chinese People's

Liberation Army, NO.55 Heping North Road, Changzhou, Jiangsu

Province 213003, China

E-mail: 13915021232@163.com

ORCID: https://orcid.org/0000-0003-2960-031X

*These authors contributed equally to this study as co-first authors. tension, which can increase the risk of cardiovascular disease [2] and mortality from cardiovascular disease [3]. Olanzapine, along with clozapine, has the greatest propensity of all available AAPs to induce weight gain and metabolic dysregulation. The underlying pathophysiological mechanisms of these metabolic disturbances have not been fully elucidated. It seems to be largely attributable to insulin resistance [4].

Metformin, as a hepatic-selective insulin sensitizer, has the potential effect of prevention or controlling the metabolic disturbance. In general population patients, metformin has widely been used to control blood glucose levels, weight gain, dyslipidemia and other metabolic dysregulation. It shows obvious effects for diabetes prevention in high-risk patients and non-diabetic obesity [5]. Accumulating evidences in the schizophrenic population have demon-

(ㄷ) This is an Open-Access article distributed under the terms of the Creative Commons Attribution Non-Commercial License (http://creativecommons.org/licenses/by-nc/4.0) which permits unrestricted non-commercial use, distribution, and reproduction in any medium, provided the original work is properly cited. 
strated that metformin is effective and safe in improving weight gain and insulin resistance induced by olanzapine [6-10]. What's more, metformin can continue to accrue with longer treatment [11]. On the other hand, metformin can improve lipid metabolic disturbances associated with the treatment of antipsychotic. A recent animal model experiment suggested that metformin could lower plasma triglycerides [12]. However, there are few literatures concerning the effect of metformin on liver fat content (LFC) during AAP therapy.

Ectopic fat storage, the storage of triglyceride droplets in nonadipose tissues (such as liver or skeletal muscle), is one of the important pathophysiological basis of insulin resistance [13], which can exacerbate hepatic insulin resistance and increase risk of developing both type 2 diabetes mellitus and cardiovascular disease [14]. The liver is a key visceral organ that is affected by ectopic fat accumulation $[15,16]$. Intrahepatic fat is associated with insulin resistance in healthy [17] and diabetic [18]. The study in overweight or obese subjects found that insulin sensitivity and cardiorespiratory fitness may be linked through intrahepatic lipid content [19]. In addition, clinical study in adults and adolescents showed that LFC, rather than total body- or visceral fat mass, can independently determine insulin resistance [20].

So far, although LFC is intimately related to insulin resistance, there have been few reports on the effect of metformin for metabolic disturbance induced by antipsychotics from the change of LFC in schizophrenia patients. In this article, we report a 16-week randomized, double-blind, placebo-controlled trial that observed the change of LFC during metformin addition for olanzapine-induced weight gain in first episode schizophrenia patients.

\section{METHODS}

\section{Design}

The study was conducted between September 2013 and September 2014. Participants were randomly assigned to treatment condition on the basis of a computergenerated sequence of numbers. Allocation was concealed using sealed opaque envelopes. The participants were randomly assigned to 16 weeks of adjunctive olanzapine with either metformin or placebo. With reference to former studies, we determined that the dose of metformin was 1,000 mg/day, which was a safe and effective dosage. For the first seven days, participants took $250 \mathrm{mg}$ of metformin or placebo three times a day. Afterwards, $500 \mathrm{mg}$ of metformin or placebo was given twice a day for rest of the trial, one before lunch and one before dinner. The olanzapine doses remained at a fixed dose as baseline levels throughout the course of treatment. After a complete description of the study to the subjects, the trial was approved by the local ethics committee and written informed consent was obtained from the subjects. The primary outcome measure was between-group change in LFC over 16 weeks. It was hypothesized that metformin would produce greater LFC reduction compared with placebo.

\section{Participants}

The participants were recruited from the No. 102 Hospital of Chinese People's Liberation Army. Eligible participants were 18 to 50 years old; met criteria for schizophrenia, as determined by the Diagnostic and Statistical Manual of Mental Disorders 4th edition (DSM-IV). All participants remained hospitalized and had the same diets throughout the trial. The same inclusion criteria were 1) under olanzapine monotherapy with a stable dose of olanzapine ( $\geq 5 \mathrm{mg} /$ day and $\leq 30 \mathrm{mg} /$ day) for at least 2 months; 2) had been maintained on the same daily doses of olanzapine for at least 4 weeks prior to study entry; 3 ) were willing to lose weight or prevent excessive body weight gain; 4) had to have relatively stable improvement (the total score of Positive and Negative Symptom Scale [PANSS] $\leq 60$ ); 5) daily alcohol consumption $<20$ g/day (men) or $10 \mathrm{~g} /$ day (women). Other enrollment criteria included that participants gained more than $7 \%$ of their predrug body weight. Participants were recruited from the first episode schizophrenia patients. All participants were asked to fill in a questionnaire about previous history and physical activities.

The following participants were excluded from the study if during the screening they had evidence of liver or renal dysfunction, cardiovascular disease, diabetes mellitus, pulmonary or neurological disease; were pregnant or lactating. Patients were also excluded if they had received a psychiatric diagnosis other than schizophrenia, or treatment with either glucose- or lipid-lowering medications, use of drugs, or any contraindication for magnetic resonance imaging (MRI).

Participants began a controlled-energy diet (near 600 kcal daily deficit) based on the American Heart Associa- 
tion recommendations that included $50 \%$ of calories from carbohydrates, $30 \%$ from fat (6\% saturated), and $20 \%$ from proteins, with a maximum cholesterol content of $300 \mathrm{mg} /$ day and $35 \mathrm{~g}$ /day of fiber. Participants were not treated with vitamins or mineral preparations during the study [21]. Participants performed light to moderate exercises for at least 30 minutes per days. The results of physical examinations and laboratory and electrocardiogram tests were normal.

All participants were informed of the study and provided written informed consent. This study was approved by the Research Ethics Review Board of No.102 Hospital of Chinese People's Liberation Army (No. 2013-015).

\section{Measures}

The baseline assessments included demographics, physical examination, including anthropometric measurements and MRI assessments. All anthropometric measures were taken by trained healthcare professionals with standardized equipment at baseline, and weeks 4, 8, 12, and 16. Anthropometric measures included weight, height, body mass index (BMI), waist circumference (WC), and waist-height ratio (WHR). WHR is thought as a better parameter of central obesity and could be applicable universally regardless of race, perhaps even gender [22]. Weight and height were measured on calibrated scales. WC was measured at the umbilicus level while the participant was standing erect. BMI was calculated as the weight $(\mathrm{kg})$ divided by the square of the height $\left(\mathrm{m}^{2}\right)$. WHR was calculated as the WC divided by the height. All body measurements were conducted after fasting for 12 hours or more.

A 1.5-T MR imager (Siemens Magnetom Essenza, Shenzhen, China) was used for MRI. Patients were asked to have breathing training before scanning in order to minimize respiration artifacts. Subjects were instructed to hold their breath for about 30 seconds each time during the scanning. Patients were examined in a supine position. Liver fat was imaged by using dual gradient echo (DGI) MRI method, according to the modified Dixon method. The entire liver was scanned twice with breath-hold (approximately for 20 seconds per scan). DGI sequence parameters were as follows: repetition time millisecond/echo time millisecond, 130/2.4 (opposed phase), 4.8 (in phase); flip angle, $70^{\circ}$; section thickness, $5.5 \mathrm{~mm}$; breath hold. MRI were visually chosen as accurately as possible on the same position in the liver. The mean signal intensity values of all regions of interest (ROIs) were determined at the same locations for in-phase and opposed-phase MRI. LFC was calculated by using the following equation [23]: $\left(\mathrm{SI}_{\mathrm{IP}}-\mathrm{Sl}_{\mathrm{OP}} / 2 \mathrm{~S} \mathrm{I}_{\mathrm{P}}\right) \times 100 \%$, where $\mathrm{SI}_{\mathrm{IP}}$ and $\mathrm{SI}_{\mathrm{OP}}$ are the mean liver signal intensity of all ROIs on in-phase and opposed-phase images, respectively. Data were processed with Imager software by an experienced magnetic resonance physicist (5 years of experience), blinded to the study results.

The primary outcomes are the changes of LFC. The secondary outcome include body weight, BMI, WC, and WHR.

\section{Statistical Methods}

Baseline characteristics of the metformin and placebo groups were compared using $t$ test for independent samples. Student's $t$ test were used as appropriate to evaluate between-group differences in changes of LFC from baseline to endpoint, and changes of body weight, BMI, WC, and WHR from baseline to each time point. The main strategy involved analysis of covariance (ANCOVA) with repeated measurements, with corresponding baseline values as covariates. Relationships between LFC and metabolic variables were assessed with Pearson or Spearman correlations. All analyses were run in SPSS, version 17.0 for Windows (SPSS Inc., Chicago, IL, USA). A $p$ value $<0.05$ was considered statistically significant. All tests were based on a two-sided level of significance.

\section{RESULTS}

\section{Demographic and Basic Descriptive Data}

Thirty-six patients entered the study. Of these, five withdrew from the study within the first 4 weeks because of exacerbation of psychosis (two placebo patient) and nausea (two metformin patients and one placebo patient). This resulted in 31 patients who were included in the study. Demographic data collected at the beginning of the study showed there was no difference between the metformin group $(n=16)$ and the placebo group $(n=15)$ in terms of age $(t=-0.28, p=0.78)$, height $(t=-1.11, p=0.28)$, weight $(t=-1.22, p=0.23)$, WC $(t=-0.58, p=0.56)$, $\operatorname{BMI}(t=-0.28, p=0.78), \mathrm{WHR}(t=0.09, p=0.78)$, and $\operatorname{LFC}(t=0.37, p=0.72)$. The two treatment groups did not differ significantly in demographic or LFC at the baseline (Table 1). 
Table 1. Baseline characteristics of participants

\begin{tabular}{lcccc}
\hline \multicolumn{1}{c}{ Variable } & Metformin group $(\mathrm{n}=16)$ & Placebo group $(\mathrm{n}=15)$ & $t$ & $p$ value \\
\hline Age $(\mathrm{yr})$ & $28.44 \pm 8.92$ & $29.27 \pm 7.44$ & -0.28 & 0.78 \\
Height $(\mathrm{m})$ & $1.61 \pm 0.09$ & $1.65 \pm 0.07$ & -1.11 & 0.28 \\
Weight $(\mathrm{kg})$ & $72.53 \pm 9.26$ & $76.47 \pm 8.65$ & -1.22 & 0.23 \\
Waist circumference $(\mathrm{m})$ & $0.95 \pm 0.11$ & $0.97 \pm 0.08$ & -0.58 & 0.56 \\
Body mass index $\left(\mathrm{kg} / \mathrm{m}^{2}\right)$ & $27.93 \pm 3.76$ & $28.28 \pm 3.33$ & -0.28 & 0.78 \\
Waist-to-height & $0.59 \pm 0.07$ & $0.59 \pm 0.06$ & 0.09 & 0.78 \\
Liver fat content $(\%)$ & $19.47 \pm 8.99$ & $18.20 \pm 10.16$ & 0.37 & 0.72 \\
\hline
\end{tabular}

Values are presented as mean \pm standard deviation.

Table 2. Liver fat content (LFC) change across the 16-week treatment between metformin group and placebo group

\begin{tabular}{lcc}
\hline \multicolumn{1}{c}{ Variable } & Metformin group & Placebo group \\
\hline LFC $(\%)$ & $16.55 \pm 7.51$ & $18.79 \pm 10.87$ \\
Difference from baseline $(\%)^{\mathrm{a}}$ & $-2.91 \pm 4.01$ & $0.59 \pm 2.87$ \\
\hline
\end{tabular}

Values are presented as mean \pm standard deviation.

${ }^{\mathrm{a}} 95 \%$ confidence interval, -6.08 to $-0.93 ; p=0.009$.

\section{Changes in LFC}

Table 2 shows descriptive data for changes in LFC over time. A two-way repeated ANOVA (drug $\times$ time) of LFC showed a significant interaction between the two factors ( $F=8.437, p<0.01)$, but not time $(F=3.956, p=0.056)$ (Fig. 1). LFC change across the 16-week treatment period was $-2.91 \%$ for the metformin group and 0.59 for the placebo group, with a between-group difference of $-3.5 \%$ (95\% confidence interval, -6.08 to $-0.93 ; p=0.009$ ) (Table 2). The between-group difference demonstrated a significant time-by-treatment interaction ( $p=0.009$ ). Table 3 shows the change from baseline to 16 weeks for primary outcome variables among weight gain inpatients with schizophrenia treated with metformin.

\section{Correlation Analysis}

The change of LFC was positively correlated with serum triglyceride level $(r=0.505, p<0.05)$ and homeostasis model assessment of insulin resistance index (HOMA-IR) level $(r=0.578, p<0.05)$ before and after metformin intervention (Table 4).

\section{DISCUSSION}

LFC is a measure of liver fat deposition. The study shows that more than $95 \%$ of liver fat exists in the form of triglycerides, and the level depends on the dynamic bal-

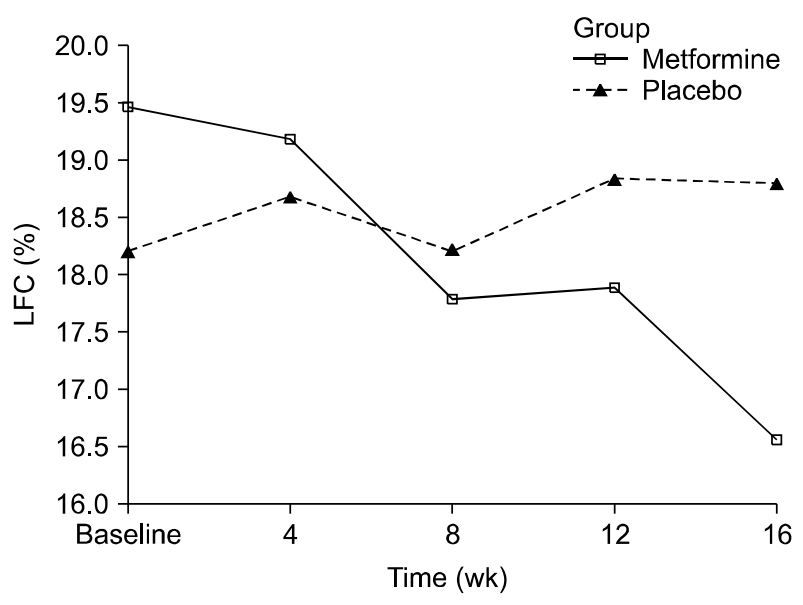

Fig. 1. Effects of 16 weeks of treatment with metformin or placebo on liver fat content (LFC). Each point represents mean \pm standard deviation based on mixed model repeated measures analysis. Time (week) effect: $F=3.956$, degree of freedom (df) $=1, p=0.056$ (non-significant); drug $\times$ time effect from mixed model analysis of variance: $F=8.437, \mathrm{df}=1, p<0.01$.

Table 3. Change from baseline to 16 weeks for primary outcome variables among weight gain inpatients with schizophrenia treated with metformin

\begin{tabular}{lcccc}
\hline \multicolumn{1}{c}{ Variable } & $\begin{array}{c}\text { Before } \\
\text { intervention }\end{array}$ & $\begin{array}{c}\text { After } \\
\text { intervention }\end{array}$ & $t$ & $p$ value \\
\hline FPG $(\mathrm{mmol} / \mathrm{L})$ & $5.3 \pm 0.2$ & $5.5 \pm 0.2$ & 0.616 & 0.543 \\
$\mathrm{HbAlc}(\%)$ & $5.0 \pm 0.6$ & $4.9 \pm 0.5$ & 0.309 & 0.762 \\
TG $(\mathrm{mmol} / \mathrm{L})$ & $2.4 \pm 0.7$ & $2.1 \pm 0.6$ & 2.242 & 0.041 \\
TC $(\mathrm{mmol} / \mathrm{L})$ & $5.3 \pm 0.6$ & $5.1 \pm 0.7$ & 1.009 & 0.329 \\
$\mathrm{HDL}-\mathrm{C}(\mathrm{mmol} / \mathrm{L})$ & $1.1 \pm 0.4$ & $1.6 \pm 0.5$ & 3.226 & 0.046 \\
LDL-C $(\mathrm{mmol} / \mathrm{L})$ & $3.1 \pm 0.4$ & $2.9 \pm 0.6$ & 1.258 & 0.228 \\
HOMA-IR & $3.5 \pm 0.6$ & $3.1 \pm 0.4$ & 2.166 & 0.047 \\
\hline
\end{tabular}

Values are presented as mean \pm standard deviation.

FPG, fasting plasma glucose; HbAlc, glycosylated hemoglobin; TG, triglycerides; TC, total cholesterol; HDL-C, high density lipoprotein cholesterol; LDL-C, low density lipoprotein cholesterol; HOMA-IR, homeostasis model assessment insulin resistance index. 
Table 4. Pearson correlation analysis of changes of LFC and glycolipid metabolism indexes before and after metformin intervention

\begin{tabular}{cccccccc}
\hline & FPG $(\mathrm{mmol} / \mathrm{L})$ & HbAlc $(\%)$ & TG $(\mathrm{mmol} / \mathrm{L})$ & $\mathrm{TC}(\mathrm{mmol} / \mathrm{L})$ & $\mathrm{HDL}-\mathrm{C}(\mathrm{mmol} / \mathrm{L})$ & $\mathrm{LDL}-\mathrm{C}(\mathrm{mmol} / \mathrm{L})$ & $\mathrm{HOMA}-\mathrm{IR}$ \\
\hline $\mathrm{LFC}(\%)$ & 0.441 & 0.378 & 0.505 & 0.450 & -0.399 & 0.286 & 0.578 \\
$p$ value & $>0.05$ & $>0.05$ & $<0.05$ & $>0.05$ & $>0.05$ & $>0.05$ & $<0.05$ \\
\hline
\end{tabular}

LFC, liver fat content; FPG, fasting plasma glucose; HbAlc, glycosylated hemoglobin; TG, triglycerides; TC, total cholesterol; HDL-C, high density lipoprotein cholesterol; LDL-C, low density lipoprotein cholesterol; HOMA-IR, homeostasis model assessment insulin resistance index.

ance between lipid intake, synthesis, output, and oxidation. Our previous studies showed that olanzapine treated patients had increased LFC, and fatty liver could occur during acute treatment. The increase of liver fat has an initiating effect on metabolic disorders of patients, and can increase the risk of diabetes and cardiovascular diseases.

Metformin was used in weight control in 1965, but the related research focused on type 2 diabetes, polycystic ovary syndrome, simple obesity and other specific groups. The effectiveness of the body weight control depends on the medium and long term treatment effect of the drug. Most of the studies have been observed for at least 12 weeks, partly for up to 4 years. In this study, the changes of LFC in patients with metformin during weight reduction were observed. The results showed that metformin intervention group could significantly reduce the LFC in the schizophrenia patients with weight gain caused by olanzapine. In the previous study of non-alcoholic fatty liver disease patients without diabetes, 24 weeks of metformin intervention could reduce the level of LFC and reach 50\% [24]. The trend of LFC in this study was consistent with this, and the mechanism may be with two metformin on adenylate activated protein kinase (AMPactivated protein kinase, AMPK) has activation effect on [25]. AMPK is regarded as the "total switch" of the body's energy. Activation of AMPK can increase the effect of glucose transporter 4, thereby increasing the insulin sensitivity of the peripheral and liver at the receptor level; at the cell level, it can reduce the synthesis of liver fat, increase the oxidation of fatty acids, reduce the accumulation of fat in the liver, and eventually reduce the fat content of the liver [26].

This study showed that the change trend of glycolipid metabolism index and insulin resistance in metformin was basically consistent with the previous research results. All of these were supported by the studies which showed metformin could significantly improve olanzapine induced metabolic disorder in schizophrenic pa- tients $[7,27,28]$. Among these indicators, there is more positive and more affirmative effect. Metformin can reduce the body weight, BMI, WC, and triglyceride level; it also has some improvement on insulin resistance [10,29], but it is not sure that the effect of metformin on the fasting blood sugar of the patient. Studies on the effects of hemoglobin are less reported.

Metformin, as a kind of guanidine oral hypoglycemic agent, has complex pharmacological effects, which can inhibit the active rotation of glucose in small intestine cells, improve the insulin sensitivity of peripheral tissue and increase the use of glucose, and improve liver insulin resistance to inhibit the liver glycogen isogenesis and reduce the liver sugar output. However, it is generally believed that metformin does not reduce the level of blood glucose in non-diabetic patients, which explains to some extent that the changes in fasting blood glucose and glycated hemoglobin are not significant in this study. In addition to hypoglycemic effect, the pharmacological action of metformin in recent years is a hot spot, which is a variety of effects of metformin and hypoglycemic effect, such as lowering serum triglyceride level and cholesterol level and increasing the level of high density lipoprotein; in some patients, the effect of reducing weight is also observed [30].

Hyperglycemia and hyperlipidemia are both the components of metabolic syndrome and the high risk factors of metabolic syndrome. Metformin has a regulatory effect on the 2. Metformin has a significant effect on blood lipids besides the well-known hypoglycemic effect. It can reduce the blood lipid level of hypertriglyceridemia to 50\%. Metformin is involved in the process of lipid metabolism by activating AMPK, regulating the process of fatty acid synthesis, oxidation and lipid lipidization, reducing the level of triglyceride [31,32], and also affecting the biosynthesis and storage of cholesterol, and reducing low density lipoprotein cholesterol and increasing the level of high density lipoprotein cholesterol. 
The results of this study showed that triglyceride levels in blood lipids were correlated with changes in LFC before and after intervention. There was a correlation between the increase of LFC and hyperlipidemia. A summary analysis of the results of a number of cross-sectional studies showed that the incidence of hyperlipidemia increased significantly with the increase of LFC and the incidence of hyperlipidemia [33]. The level of lipoprotein cholesterol, in the large sample study of two Chinese people, the LFC and serum triglyceride level $(r=0.260$ and $0.263)$, high density lipoprotein cholesterol level $(r=-0.320$ and -0.147 , respectively) were related. The result of this study is close to that of it. The results of one study in the animal model of fatty liver provide more direct evidence for the results of this study. The study showed that metformin can effectively reduce the LFC and the expression of liver fatty acid synthetase. Meanwhile the decrease rate of LFC was positively correlated with the decrease rate of triglyceride, total abdominal fat and insulin level [32].

No correlation was found between LFC and fasting blood glucose and glycosylated hemoglobin. We consider a variety of factors that may affect the results of correlation analysis: 1) no significant changes in glycometabolism before and after metformin intervention were observed in this study; 2) previous studies have not been clear about the relationship between glycometabolism and LFC. One study reported that there was a significant correlation between them $(r=0.599)$, but a later study found that the correlation between the two groups was not significant $(r=$ 0.18). We further analyzed the two results and found that the previous study included a considerable proportion (62/109) of patients with abnormal glucose metabolism. The relationship between blood glucose and LFC was different in the latter study. It is not difficult to understand that the blood glucose level of this group is in the normal range and is closer to the later subjects.

It should be pointed out that this result does not explain the causal relationship between the changes of LFC and the changes of insulin resistance after metformin treatment, but can only reflect the both of them are coexistence and interrelated components of metformin intervention. Which one is first, LFC or insulin resistance? Some researchers argued that liver fat deposition is the consequence of insulin resistance [34], and other researchers believed that liver fat deposition has a starting effect on insulin resistance. However, more viewpoints hold that the two factors are mutual cause and effect, forming a vicious circle, forming the common foundation of the pathogenesis of metabolic syndrome. In explaining the correlation between LFC and insulin resistance after metformin intervention, we also have a compromise view due to lack of sufficient evidence.

The main shortcomings of this study are as follows. First, the small sample size can affect the efficiency of statistics; because of the existence of gender bias, it has an impact on some of the research indicators. Second, many indicators of this study, including LFC, are affected by many factors such as age, sex and so on. It is difficult to correct them effectively because of the small sample size. In addition, the determination of LFC by MRI is technically demanding and expensive, and is not suitable for clinical application. In the follow-up study, ultrasound LFC quantification technology can be considered as a substitute for clinical application.

Despite these limitations, the results from this study have provided preliminary evidence that metformin is effective in promoting reducing LFC and weight gain induced by olanzapine in patients with first episode schizophrenia. In the process of metformin intervention in the schizophrenic patients with olanzapine induced weight gain, LFC decreased, accompanied by significant changes in glycolipid metabolism index and insulin resistance, and correlated with the changes of LFC to varying degrees. This study has provided some insight in this area, but much additional work remains, and important advances are anticipated in the coming years.

\section{- Conflicts of Interest}

No potential conflict of interest relevant to this article was reported.

\section{- Author Contributions}

Conceptualization: Li Wang, Yu Chen, Le-ping Xu. Data acquisition: Li Wang, Yun-chuan Sui, Zhi Zhou. Formal analysis: Le-ping $\mathrm{Xu}$, Xing-qi Tan. Funding: Xing-qi Tan. Supervision: Le-ping Xu, Ning Li. Writingoriginal draft: Yu Chen, Li Wang. Writing-review \& editing: Li Wang, Yu Chen.

\section{ORCID}

Yu Chen https://orcid.org/0000-0003-2722-9561 
Yun-chuan Sui

https://orcid.org/0000-0002-3013-3512

Xing-qi Tan

https://orcid.org/0000-0002-7001-3662

Zhi Zhou

Ning Li

Le-ping Xu

https://orcid.org/0000-0003-4671-9240

https://orcid.org/0000-0003-2973-4350

https://orcid.org/0000-0003-2960-031X

\section{REFERENCES}

1. Coccurello R, Moles A. Potential mechanisms of atypical antipsychotic-induced metabolic derangement: clues for understanding obesity and novel drug design. Pharmacol Ther 2010;127:210-251.

2. De Hert M, Detraux J, van Winkel R, Yu W, Correll CU. Metabolic and cardiovascular adverse effects associated with antipsychotic drugs. Nat Rev Endocrinol 2011;8:114-126.

3. Henderson DC, Nguyen DD, Copeland PM, Hayden DL, Borba CP, Louie PM, et al. Clozapine, diabetes mellitus, hyperlipidemia, and cardiovascular risks and mortality: results of a 10-year naturalistic study. J Clin Psychiatry 2005;66: 1116-1121.

4. Eckel RH, Grundy SM, Zimmet PZ. The metabolic syndrome. Lancet 2005;365:1415-1428.

5. Howland RH. A "glucose eater" drug as a therapeutic agent in psychiatry. J Psychosoc Nurs Ment Health Serv 2013;51:1316.

6. Wang M, Tong JH, Zhu G, Liang GM, Yan HF, Wang XZ. Metformin for treatment of antipsychotic-induced weight gain: a randomized, placebo-controlled study. Schizophr Res 2012;138:54-57.

7. Wu RR, Jin H, Gao K, Twamley EW, Ou JJ, Shao P, et al. Metformin for treatment of antipsychotic-induced amenorrhea and weight gain in women with first-episode schizophrenia: a double-blind, randomized, placebo-controlled study. Am J Psychiatry 2012;169:813-821.

8. Ehret M, Goethe J, Lanosa M, Coleman Cl. The effect of metformin on anthropometrics and insulin resistance in patients receiving atypical antipsychotic agents: a meta-analysis. J Clin Psychiatry 2010;71:1286-1292.

9. Praharaj SK, Jana AK, Goyal N, Sinha VK. Metformin for olanzapine-induced weight gain: a systematic review and metaanalysis. Br J Clin Pharmacol 2011;71:377-382.

10. Newall H, Myles N, Ward PB, Samaras K, Shiers D, Curtis J. Efficacy of metformin for prevention of weight gain in psychiatric populations: a review. Int Clin Psychopharmacol 2012; 27:69-75.

11. Jarskog LF, Hamer RM, Catellier DJ, Stewart DD, Lavange L, Ray N, et al. Metformin for weight loss and metabolic control in overweight outpatients with schizophrenia and schizoaffective disorder. Am J Psychiatry 2013;170:1032-1040.

12. Geerling JJ, Boon MR, van der Zon GC, van den Berg SA, van den Hoek AM, Lombès M, et al. Metformin lowers plasma triglycerides by promoting VLDL-triglyceride clearance by brown adipose tissue in mice. Diabetes 2014;63:880-891.
13. D’Adamo E, Cali AM, Weiss R, Santoro N, Pierpont B, Northrup $\mathrm{V}$, et al. Central role of fatty liver in the pathogenesis of insulin resistance in obese adolescents. Diabetes Care 2010;33:1817-1822.

14. Byrne CD, Targher G. Ectopic fat, insulin resistance, and nonalcoholic fatty liver disease: implications for cardiovascular disease. Arterioscler Thromb Vasc Biol 2014;34:1155-1161.

15. Byrne CD. Ectopic fat, insulin resistance and non-alcoholic fatty liver disease. Proc Nutr Soc 2013;72:412-419.

16. Byrne CD. Dorothy Hodgkin Lecture 2012: non-alcoholic fatty liver disease, insulin resistance and ectopic fat: a new problem in diabetes management. Diabet Med 2012;29:10981107.

17. Chan DC, Watts GF, Ng TW, Hua J, Song S, Barrett PH. Measurement of liver fat by magnetic resonance imaging: relationships with body fat distribution, insulin sensitivity and plasma lipids in healthy men. Diabetes Obes Metab 2006;8: 698-702.

18. Kotronen A, Juurinen L, Tiikkainen M, Vehkavaara S, Yki-Järvinen $\mathrm{H}$. Increased liver fat, impaired insulin clearance, and hepatic and adipose tissue insulin resistance in type 2 diabetes. Gastroenterology 2008;135:122-130.

19. Haufe S, Engeli S, Budziarek P, Utz W, Schulz-Menger J, Hermsdorf M, et al. Cardiorespiratory fitness and insulin sensitivity in overweight or obese subjects may be linked through intrahepatic lipid content. Diabetes 2010;59:1640-1647.

20. Linder K, Springer F, Machann J, Schick F, Fritsche A, Häring $\mathrm{HU}$, et al. Relationships of body composition and liver fat content with insulin resistance in obesity-matched adolescents and adults. Obesity (Silver Spring) 2014;22:1325-1331.

21. Lichtenstein AH, Appel LJ, Brands M, Carnethon M, Daniels S, Franch HA, et al. Summary of American Heart Association diet and lifestyle recommendations revision 2006. Arterioscler Thromb Vasc Biol 2006;26:2186-2191.

22. Parikh RM, Joshi SR, Menon PS, Shah NS. Index of central obesity - a novel parameter. Med Hypotheses 2007;68:12721275.

23. Cassidy FH, Yokoo T, Aganovic L, Hanna RF, Bydder M, Middleton MS, et al. Fatty liver disease: MR imaging techniques for the detection and quantification of liver steatosis. Radiographics 2009;29:231-260.

24. Bugianesi E, Gentilcore E, Manini R, Natale S, Vanni E, Villanova $\mathrm{N}$, et al. A randomized controlled trial of metformin versus vitamin Eor prescriptive diet in nonalcoholic fatty liver disease. Am J Gastroenterol 2005;100:1082-1090.

25. Yang J, Holman GD. Long-term metformin treatment stimulates cardiomyocyte glucose transport through an AMP-activated protein kinase-dependent reduction in GLUT4 endocytosis. Endocrinology 2006;147:2728-2736.

26. Rector RS, Thyfault JP, Wei Y, Ibdah JA. Non-alcoholic fatty liver disease and the metabolic syndrome: an update. World J Gastroenterol 2008;14:185-192.

27. Chen $\mathrm{CH}$, Chiu CC, Huang MC, Wu TH, Liu HC, Lu ML. 
Metformin for metabolic dysregulation in schizophrenic patients treated with olanzapine. Prog Neuropsychopharmacol Biol Psychiatry 2008;32:925-931.

28. Ellinger LK, Ipema HJ, Stachnik JM. Efficacy of metformin and topiramate in prevention and treatment of second-generation antipsychotic-induced weight gain. Ann Pharmacother 2010; 44:668-679.

29. Maayan L, Correll CU. Management of antipsychotic-related weight gain. Expert Rev Neurother 2010;10:1175-1200.

30. Wulffelé MG, Kooy A, de Zeeuw D, Stehouwer CD, Gansevoort RT. The effect of metformin on blood pressure, plasma cholesterol and triglycerides in type 2 diabetes mellitus: a systematic review. J Intern Med 2004;256:1-14.

31. Li Y, Xu S, Mihaylova MM, Zheng B, Hou X, Jiang B, et al. AMPK phosphorylates and inhibits SREBP activity to attenuate hepatic steatosis and atherosclerosis in diet-induced insulin-resistant mice. Cell Metab 2011;13:376-388.

32. Chen WL, Wei HW, Chiu WZ, Kang CH, Lin TH, Hung CC, et al. Metformin regulates hepatic lipid metabolism through activating AMP-activated protein kinase and inducing ATGL in laying hens. Eur J Pharmacol 2011;671:107-112.

33. Isla Pera P; American Diabetes Association. [Chronic complications of diabetes mellitus. Recommendations from the American Diabetes Association 2011. Prevention and management]. Rev Enferm 2012;35:46-52. Spanish.

34. Targher G, Bertolini L, Padovani R, Rodella S, Tessari R, Zenari $\mathrm{L}$, et al. Prevalence of nonalcoholic fatty liver disease and its association with cardiovascular disease among type 2 diabetic patients. Diabetes Care 2007;30:1212-1218. 